\title{
General management strategies for efavirenz therapy and associated adverse events*
}

\author{
Jean-Guy Baril MD¹, Anita Rachlis MD MEd FRCPC ${ }^{2}$
}

J-G Baril, A Rachlis. General management strategies for efavirenz therapy and associated adverse events. Can J Infect Dis Med Microbiol 2006;17(Suppl D):10D-14D.

Efavirenz therapy-related central nervous system symptoms often occur shortly after initiation of treatment; therefore, the timing of therapy initiation must be carefully considered so that if side effects do occur, it is at a time when the patient is not mentally overburdened and when support is readily available. Current literature contains contradictory data on the relationship between plasma levels of efavirenz and the occurrence of neuropsychiatric adverse events; therefore, routine measurement of plasma concentrations is not recommended except under certain circumstances. Clinician management of general neuropsychiatric symptoms comprises four steps: preparation, education, reassurance and treatment. Among the specific central nervous system symptoms that are known to occur with efavirenz therapy are agitation, sleep disturbances, dreams, dizziness, impaired concentration and depression. There are recommended pharmacological and nonpharmacological protocols for managing these symptoms. Prompt, successful management of treatment-emergent neuropsychiatric symptoms is important so that the patient may receive the optimal longterm viral suppression that is possible with efavirenz therapy.

Key Words: Adverse events; Central nervous system; Efavirenz; Neuropsychiatric; Side effects

Recause central nervous system (CNS) symptoms may appear Bwithin the first or second day of therapy, efavirenz should ideally be initiated during a period that will not require the patient's focused attention or energy, and during which the patient has ready access to support. The clinician must work with the patient to determine the optimal timing for efavirenz initiation, recognizing the possible adverse events (AEs) and the strategies used to manage these potential AEs. Therefore, intensive education before initiation of therapy, ongoing patient support and close monitoring are essential elements affecting a patient's ability to achieve maximum therapeutic benefit from efavirenz.

\section{GENERAL RECOMMENDATIONS}

Bartlett et al (1) recommend the following four-step approach for managing neuropsychiatric side effects on initiation of efavirenz therapy.

\section{Prepare}

- Ensure that the patient is ready to start highly active antiretroviral therapy.

- Screen and stabilize pre-existing neuropsychiatric symptoms.

\section{Stratégies de prise en charge globale du traite- ment à l'éfavirenz et de ses effets indésirables}

Les symptômes d'origine nerveuse centrale, liés à l'éfavirenz apparaissent habituellement peu de temps après l'instauration du traitement; aussi importe-t-il de bien choisir le moment de la mise en route du traitement de sorte que, s'il y a manifestation de symptômes, le patient ne soit pas trop occupé mentalement et qu'il ait accès rapidement à du soutien. La documentation actuelle contient des données contradictoires sur le lien entre la concentration plasmatique d'éfavirenz et l'apparition d'effets indésirables psychiatriques; il n'est donc pas recommandé d'effectuer des mesures systématiques de la concentration plasmatique d'éfavirenz, sauf cas particuliers. La prise en charge globale des symptômes neuropsychiatriques comprend quatre étapes : la préparation, l'éducation, la rassurance et le traitement. Parmi les symptômes d'origine nerveuse centrale, typiquement liés à l'éfavirenz, mentionnons l'agitation, les troubles du sommeil, les rêves, les étourdissements, le manque de concentration et la dépression. Il existe des protocoles de traitement médicamenteux et non médicamenteux contenant des recommandations sur la prise en charge de ces symptômes. Il est important de traiter rapidement et convenablement l'apparition des symptômes neuropsychiatriques pour que les patients puissent profiter le plus longtemps possible de la suppression virale produite par le traitement à l'éfavirenz.
- If the patient has a history of psychiatric illnesses, establish or re-establish support systems and mental health contact.

- Stabilize and treat any identified conditions using standard psychiatric interventions.

Educate

- Discuss the most common neuropsychiatric side effects.

- Provide counselling and ensure that the patient understands the common potential AEs of drug therapy, including their nature, frequency, severity and duration.

- Provide written information pertaining to AEs, when available.

\section{Reassure}

- Convey important messages that aid in therapy compliance.

$\circ$ Efavirenz is effective for HIV.

- The neuropsychiatric side effects of efavirenz are in the mild to moderate range, are time limited and result in few discontinuations.

${ }^{1}$ Clinique Médicale du Quartier Latin and Service-Consultation-Liaison VIH/SIDA, CHUM (University of Montreal Hospital Centre), Montreal, Quebec; ${ }^{2}$ Division of Infectious Diseases, Department of Medicine, Sunnybrook Health Sciences Centre, University of Toronto, Toronto, Ontario

*This article is a 2006 update of the following previously published 2001 article: MJ Gill, A Rachlis, S Walmsley, M Halman and the Efavirenz Consensus Working Group. Canadian Expert Panel recommendations on the management of CNS symptoms related to efavirenz. Can J Infect Dis 2001;12(Suppl C):20C-30C.

Correspondence: Dr Jean-Guy Baril, 905 Rene Levesque Boulevard Est, Montreal, Quebec H2L 5B1. Telephone 514-285-5500, fax 514-285-2226, e-mail jgbaril@videotron.ca 
- During the initial period of efavirenz therapy, provide close patient follow-up or monitoring.

- In some cases, consider a program in which the patient is contacted within a few days of initiating efavirenz therapy.

- Ensure that patient support is available.

Treat

- Address new-onset and persistent neuropsychiatric symptoms.

- Continue patient support and monitoring.

- Determine any other causes or exacerbating factors for the symptoms experienced.

- Evaluate the severity of symptoms and their impact on the patient's quality of life.

- Treat emergent neuropsychiatric syndromes with standard psychiatric interventions.

- If symptoms persist despite psychiatric intervention, discontinuing efavirenz may be necessary.

\section{MANAGEMENT RECOMMENDATIONS FOR SPECIFIC CNS SYMPTOMS ASSOCIATED WITH EFAVIRENZ}

Most of the following recommendations for CNS symptoms are based on clinical experience and judgement. There are no clinical trials or prospective evaluations for these recommendations in HIV populations receiving efavirenz. These recommendations are derived from consensus meetings of clinicians and from published expert recommendations (1-5).

The literature contains contradictory data on the relationship between plasma levels of efavirenz and the occurrence of neuropsychiatric AEs. Two studies $(6,7)$ and at least one anecdotal report (8) indicate that these AEs occur more frequently in cases of high plasma levels of efavirenz. Conversely, other studies (9-11) found no correlation between plasma levels of efavirenz and neuropsychiatric disorders. Research, particularly in pharmacogenetics, is currently underway to further investigate the nature of this relationship. An ACTG 5095 substudy (12) compared the different genotype variants of cytochromes involved in efavirenz metabolism. It appears that some CYP2B6 and CYP3A4 genotypes were associated with high plasma levels of efavirenz, with only the CYP2B6 genotype associated with neuropsychiatric AEs (12). Considering the conflicting data, measurement of plasma levels of efavirenz is not recommended under normal circumstances; however, monitoring may be useful in some cases of persistent side effects (8). Additional studies must be performed to further investigate this area.

Certain CNS-specific, efavirenz-associated symptoms, including agitation, sleep disturbances, dreams, dizziness, impaired concentration and depression, have a recommended management protocol (Table 1).

\section{Agitation}

Agitation is one of the CNS symptoms that may be experienced by patients on efavirenz. However, agitation may also be due to other underlying etiologies, such as major depression or anxiety over new stressors in a patient's life, or secondary to pharmacological agents such as caffeine, prednisone, antidepressants with activating AEs and substance abuse (13-15).
Once other causes of agitation have been ruled out, ongoing supportive counselling to encourage continuation of therapy (because this symptom is likely to resolve over time) is essential. Further strategies may include stress-relieving activities; empirical pharmacological treatment that has been carefully selected to avoid drug interactions (16); benzodiazepines or neuroleptic agents (haloperidol, clozapine, olanzapine), which may be effective in the management of agitation and aggression in the nonHIV psychiatric patient population $(13,15)$; and anxiolytics (see Table 1). Because anxiolytics are potentially addictive, such drugs should be used only for acute agitation and their use should be limited to no more than four to eight weeks. Although antipsychotic medications are not indicated for the management of agitation, some patients with nonpsychotic disorders presenting with agitation may respond to a low-dose neuroleptic while awaiting a formal psychiatric evaluation (Table 1).

\section{Sleep disturbances}

Insomnia and sleep disturbances are widespread and often underdiagnosed in HIV-positive patients. In one study (17), $73 \%$ of the HIV patients were found to have insomnia, yet only $45 \%$ of the patient charts contained any documentation of insomnia. Insomnia may be caused by many factors: psychiatric (depression, anxiety), medical (sleep apnea, pulmonary disease, HIV/AIDS) and pharmacological (caffeine, alcohol, secondary to psychotropics including antidepressants). Causes of insomnia should be explored and treated appropriately.

Nonpharmacological behavioural strategies can be used to manage sleep disturbances $(17,18)$ (Table 1). Sleep hygiene measures (Table 2) aim to remove possible deterrents to sleep, to apply structure to sleep patterns and to prevent association of the bed with activities of wakefulness. Additional interventions, such as progressive muscle relaxation, biofeedback and cognitive behavioural therapy, may be beneficial. Anecdotally, adjusting the administration times of efavirenz to earlier in the evening or during the day has also provided symptom relief.

Pharmacological therapy may include the use of hypnotics, including short-acting benzodiazepines (ie, lorazepam or oxazepam) or the nonbenzodiazepine hypnotic zopiclone (Table 1). These agents may be particularly useful in patients who have early insomnia (trouble falling asleep). For patients suffering from middle insomnia (trouble maintaining sleep), a longer-acting hypnotic (eg, low-dose trazodone) may be considered. Long-acting benzodiazepines have active metabolites that produce more AEs, including cognitive side effects, and have a higher potential for drug interaction in patients with HIV/AIDS (14). In addition, concurrent administration of protease inhibitors with benzodiazepines that are substrates of CYP3A4 (eg, diazepam) may result in an increased benzodiazepine concentration and additional AEs $(19,20)$. Terminal insomnia (early morning awakening) is a classic sign of major depression, and patients presenting with it should be evaluated for major depression and treated accordingly. Often, sleep disturbances and major depression require treatment with the use of an antidepressant and a hypnotic. Nevertheless, balancing the need for sleep and the potential toxicities associated with long-term hypnotic usage is challenging. Generally, hypnotics should be used at the lowest feasible dose and for as limited a duration as possible. Prescriptions should be monitored and small amounts prescribed to mitigate against nonjudicious usage patterns. Failure to manage sleep symptoms should prompt a psychiatric consultation or evaluation by a sleep specialist. 
TABLE 1

Symptom-specific management strategies for HIV-positive patients taking efavirenz

Initial period of efavirenz therapy

\begin{tabular}{|c|c|}
\hline $\begin{array}{l}\text { Specific } \\
\text { symptom }\end{array}$ & Nonpharmacological \\
\hline \multirow[t]{3}{*}{ Agitation } & $\begin{array}{l}\text { Evaluate for other potential causes, including } \\
\text { psychiatric and medical causes, and substance use } \\
\text { and manage accordingly }\end{array}$ \\
\hline & $\begin{array}{l}\text { Minimize stimulant intake, including caffeine, } \\
\text { prescription or over-the-counter } \\
\text { medications, or illegal substances }\end{array}$ \\
\hline & $\begin{array}{l}\text { Suggest stress-relieving activities } \\
\text { (eg, exercise, meditation, relaxation techniques) }\end{array}$ \\
\hline \multirow[t]{2}{*}{$\begin{array}{l}\text { Sleep } \\
\text { disturbances }\end{array}$} & $\begin{array}{l}\text { Evaluate for underlying cause of insomnia } \\
\text { and treat accordingly }\end{array}$ \\
\hline & Evaluate patient's sleep hygiene practices (Table 2) \\
\hline
\end{tabular}

Dreams

Consider exercises such as focused thoughts, visualization and the use of soft music before sleep

Maintain regular sleep patterns

Before going to bed, relax or engage in pleasant activities (eg, soft music, pleasant movies), and avoid scary or violent movies, television programs or books

Sleep with a nightlight, if helpful

Pharmacological

Persistent symptoms

Anxiolytics for mild agitation, such as lorazepam

$0.5 \mathrm{mg}$ to $2 \mathrm{mg}$ every $12 \mathrm{~h}$ as needed or

Psychiatric consultation clonazepam $0.5 \mathrm{mg}$ to $1 \mathrm{mg}$ every $12 \mathrm{~h}$ as needed. agitation

Daily doses should not exceed $4 \mathrm{mg}$, and use should be limited to four to eight weeks' duration

For more severe cases, consider a low-dose neuroleptic such as olanzapine (Zyprexa, Eli Lilly Canada Inc) $2.5 \mathrm{mg}$ to $5 \mathrm{mg}$ at bedtime pending a more formal psychiatric evaluation

Adjust efavirenz dosage administration time earlier (eg, during the day)

Early insomnia: use short-acting benzodiazepine, such as lorazepam $1 \mathrm{mg}$ to $2 \mathrm{mg}$ at bedtime as needed, and consider giving a short prescription (ie, no more than two weeks) at the time of efavirenz initiation

Middle insomnia: a longer-acting hypnotic may be required. Low-dose trazodone (Desyrel, Bristol Myers-Squibb, Canada), $25 \mathrm{mg}$ to $100 \mathrm{mg}$ at bedtime, is recommended

Terminal insomnia: the patient should be assessed for depression and treated as appropriate. An antidepressant with hypnotic effect, such as low-dose trazodone, $25 \mathrm{mg}$ to $100 \mathrm{mg}$ at bedtime, is recommended

Consider efavirenz dosing administration adjustment (earlier in the evening or during the day, or twice-daily administration with a larger dose at bedtime

Consider a trial of short-acting benzodiazepine, such as lorazepam $1 \mathrm{mg}$ to $2 \mathrm{mg}$ at bedtime as needed

For more severe cases, other options include:

- low-dose (1 mg) haloperidol

- low-dose (2.5 mg) olanzapine (Zyprexa)

- low-dose (25 mg to $100 \mathrm{mg}$ ) trazodone (Desyrel)

Dizziness If the dizziness is persistent or atypical, screen for other causes:

- ear, nose and throat disorders (eg, ear or sinus infections)

- any other medical or psychiatric condition that may cause dizziness

Counsel patients on avoiding activities requiring concentration (eg, driving a car)

Counsel patients on home environment safety. Identify and remove any obstacles (eg, clothing on floor) that may cause accidental falls

Impaired Try to start efavirenz when the patient can expect to have concentration minimal mental demands (eg, on the weekend) When starting efavirenz, limit mentally demanding activity initially (eg, avoid multitasking and situations that require intense concentration)

Proper use of caffeine-based beverages, such as coffee or colas, may be helpful

Depression Early psychiatric treatment: re-establish patient contact with psychiatric care if a patient has a history, and, if needed, initiate a psychiatric consult for patients with a new episode

Provide psychosocial support and structured therapy

Pay special attention to issues of patient safety

Ensure that patient is aware of and has access to crisis support services

Reconsider efavirenz dose schedule to allow $12 \mathrm{~h}$ to $15 \mathrm{~h}$ between the dose and when the patient usually experiences dizziness (eg, if the patient experiences dizziness in the mornings, try changing the dose from bedtime to dinnertime)

Consider twice-daily dosing, with a smaller dose at bedtime

Rule out concurrent psychoactive drugs, including illicit drugs

Meclizine (Bonamine, Pfizer Canada Inc)

Dimenhydrinate (Gravol, Church \& Dwight Canada Corp)

Consider adjustment to efavirenz dosing administration (depending on when symptom is most severe)

A trial of twice-daily administration may be considered

Prescribe antidepressant therapy for patients with moderate to severe symptoms. An antidepressant (preferably a selective serotonin reuptake inhibitor) alone or in combination with psychotherapy should be prescribed. Initiate at a low dose and titrate slowly
Psychiatric consultation

Evaluation by a sleep specialist
Use memory aids as used in neurorehabilitation (eg, memory book or sticky notes)

Continue to evaluate for other potential causes, such as depression, and treat as appropriate

Refer to a psychiatrist If symptoms do not respond to standard psychiatric intervention, consider a change in antiretroviral combination

Provide psychotherapy and psychosocial support for mild to moderate symptoms

See Table 3 for patient counselling strategies 
TABLE 2

\section{Recommended strategies for sleep hygiene}

Avoid caffeine, chocolate, nicotine and alcohol before bedtime, and try to limit consumption of these substances during the day

Practice good sleep habits (ie, going to bed at the same time each night and waking up at the same time every day)

Use the bed for sleep and sex only (ie, not for watching television or working)

Try relaxation techniques before bedtime (eg, a warm bath, yoga or breathing exercises)

Exercise in the mornings instead of the evenings

\section{Dreams}

Many patients taking efavirenz report vivid dreams. Dream content varies widely, from pleasant to disturbing in nature. Some patients complain of dreams so vivid that they do not get a restful sleep. No studies have been undertaken on the management of disturbing dreams in either the HIV or general patient population. Patient education before initiating treatment and ongoing supportive counselling are critical to successful therapy.

Nonpharmacological and pharmacological interventions that have anecdotally provided relief are included in Table 1.

\section{Dizziness}

Dizziness is often described as light-headedness and a feeling of unsteadiness (ie, unsure of where to put one's feet). Patients may experience dizziness at any time of the day. Supportive counselling remains the mainstay of dizziness management. However, the clinician should rule out other causes of dizziness, such as concurrent psychoactive drugs.

Anecdotal reports of adjusting the timing of efavirenz administration to minimize symptoms have resulted in symptom improvement; more nonpharmacological and pharmacological interventions that may help manage dizziness are included in Table 1. Patients with severe dizziness and those at risk of falls should be reassessed regarding therapeutic options.

\section{Impaired concentration}

Impaired concentration is one of the efavirenz-related CNS symptoms that usually resolves within a few weeks (21). In addition to reassurance and supportive counselling, the physician should assess the patient for underlying causes of impaired concentration, such as insomnia, depression, cognitive disorders associated with HIV/AIDS and substance-related disorders (22-24).

Anecdotally, adjusting the dosing administration of efavirenz according to the time during the day when impaired concentration occurs or is most severe has provided symptom relief. Splitting the dose to a twice-daily administration has also been tried. In addition, coping tactics should be discussed with patients who have persistent impaired concentration, such as neurorehabilitation strategies using prompting and compensatory techniques (Table 1), including the use of a memory book, sticky notes, a computer or paging devices to reduce the patient's reliance on memory.

\section{Depression}

Depressive symptoms (eg, anxiety, agitation, insomnia) are common in patients with HIV/AIDS and may be due to many factors, including a disposition to major depression, psychosocial stressors, progression of HIV/AIDS, psychoactive substance
TABLE 3

Patient counselling strategies for depression

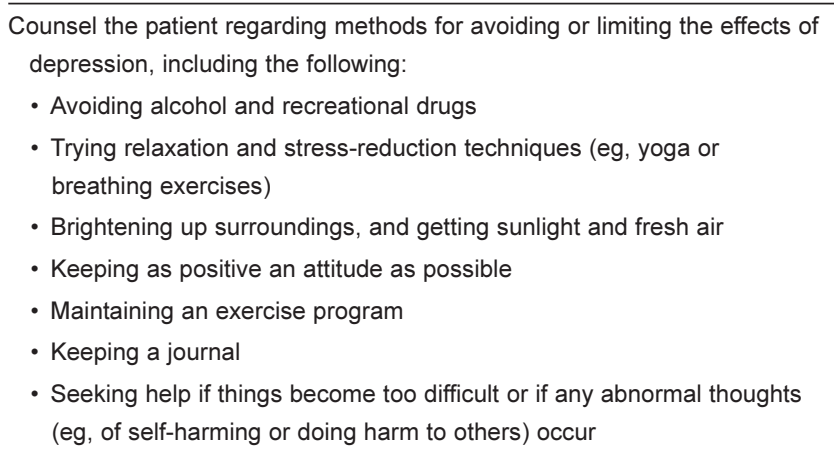

abuse or dependence, or a side effect of medications, such as efavirenz, zidovudine, interferon and prednisone (24).

Early identification of depression and commencement of treatment is important. Screening questions with appropriate follow-up should be incorporated into the routine monitoring by clinic staff (see Halman, pages 5D-9D). The diagnosis of major depression is made by identifying the classic symptoms of major depression as outlined in the Diagnostic and Statistical Manual of Mental Disorders, Fourth Edition (25). Major depression in patients with HIV/AIDS is managed using standard psychiatric interventions, and these are useful regardless of the presumed underlying cause of the major depression. It is also recommended that patients be counselled on strategies that can help them limit or avoid the effects of depression (Table 3).

In patients with mild to moderate depressive symptomatology, psychotherapy alone may help remit symptoms. In patients with moderate to severe symptoms, psychopharmacological intervention alone or in combination with psychotherapy is necessary. Antidepressants such as selective serotonin reuptake inhibitors and tricyclic antidepressants have demonstrated similar efficacy in HIV patients as in the general population (26-29). Selective serotonin reuptake inhibitors are generally better tolerated agents and have emerged as first-line interventions (30).

Antidepressant treatment should be initiated at a low dose and titrated slowly to standard therapeutic doses (11). Patients should be initiated on one-half of the recommended starting dose and titrated to the starting dose after seven to 10 days if the drug is well tolerated. The patient's clinical response should be evaluated regularly, paying special attention to patient safety and drug tolerability, with the dosage adjusted accordingly (31). Response typically begins to occur after three weeks following treatment initiation, with significant improvement generally seen by weeks 4 to 6 . A lack of response to one agent at week 6 should prompt a dosage increase using a standard augmentation strategy, or, if possible, a switch to a second agent. Psychiatric evaluation could be sought, particularly in patients who do not respond to standard interventions. After the remission of depression is achieved, it is necessary to continue therapy for at least nine to 12 months to consolidate the effect and minimize relapse (22).

\section{CONCLUSIONS}

Medication AEs are frequently cited as the reasons for patients' therapy interruption or treatment noncompliance. Having established strategies and protocols in place to address AEs may 
help mitigate their impact, allowing patients to continue on their therapy and maximize their treatment outcomes.

A significant number of efavirenz-treated patients experience CNS symptoms. The present paper outlined general management recommendations for any AE symptom related to efavirenz treatment initiation, as well as specific management strategies for CNS symptoms, including agitation, sleep disturbances, dreams, dizziness, impaired concentration and

\section{REFERENCES}

1. Bartlett JA, Ferrando SJ. Identification and management of neurologic and psychiatric side effects associated with HIV and HAART. <http://www.medscape.com/viewarticle/470017_1>

2. Gill MJ, Rachlis A, Walmsley S, Halman M; Efavirenz Consensus Working Group. Canadian Expert Panel recommendations on the management of CNS symptoms related to efavirenz. Can J Infect Dis 2001;12(Suppl C)20C-30C.

3. Bary M, David F, Gasnault C, et al. [Troubles neuropsychiatriques chez les patients infectés par le VIH et rôle de l'efavirenz.] Méd Mal Infect 2004;34:435-49.

4. Clifford DB, Evans S, Yang Y, et al. Impact of efavirenz on neuropsychological performance and symptoms in HIV-infected individuals. Ann Intern Med 2005;143:714-21.

5. HIV and Hepatitis.com. Managing Sustiva (efavirenz) Side Effects. $<$ http://www.hivandhepatitis.com/hiv_and_aids/sustivasideeffects $4 . h$ tm l\#one> (Version current on October 6, 2006).

6. Marzolini C, Telenti A, Decosterd LA, Greub G, Biollaz J, Buclin T. Efavirenz plasma levels can predict treatment failure and central nervous system side effects in HIV-1-infected patients. AIDS 2001;15:71-5.

7. Nunez M, Gonzalez De Requena D, Gallego L, Jimenez-Nacher I, Gonzalez-Lahoz J, Soriano V. Higher efavirenz plasma levels correlate with development of insomnia. J Acquir Immune Defic Syndr 2001;28:399-400

8. Hasse B, Gunthard HF, Bleiber G, Krause M. Efavirenz intoxication due to slow hepatic metabolism. Clin Infect Dis 2005;40:e22-3.

9. Fiske WD, Joshi AS, Labriola DF. An assessment of population pharmacokinetic parameters of efavirenz on nervous system symptoms and suppression of HIV-RNA. 41st Interscience Conference on Antimicrobial Agents and Chemotherapy. Chicago, September 22 to 25, 2001. (Abst A-1727)

10. Raspall T, Blanco J, Blanch J. Neuropsychological disturbances and PK levels in patients receiving efavirenz: A pilot study. 4th International Workshop on Clinical Pharmacology of HIV Therapy. Cannes, March 27 to 29, 2003. (Abst)

11. Ribaudo H, Clifford D, Gulick R, et al. Relationship between efavirenz pharmacokinetics, side effects, drug discontinuation, virologic response, and race: Results from ACTG A5095/A5097s. Foundation for Retrovirology and Human Health. 11th Conference on Retroviruses and Opportunistic Infections. San Francisco, February 8 to 11,2004 . (Abst)

12. Haas D, Ribaudo H, Kim R, et al. A common CYP2B6 variant is associated with efavirenz pharmacokinetics and central nervous system side effects: AACTG Study NWCS214. Foundation for Retrovirology and Human Health. 11th Conference on Retroviruses and Opportunistic Infections. San Francisco, February 8 to 11, 2004. (Abst)

13. Buckley PF. The role of typical and atypical antipsychotic medications in the management of agitation and aggression. J Clin Psychiatry 1999;60(Suppl 10):52-60. depression. Nonpharmacological and pharmacological interventions are included both for treatment-emergent symptoms that may occur on therapy initiation and for ongoing symptoms. We hope that these recommendations will help the clinician provide patient support in the event of treatment-emergent AEs so that the patient may receive the optimal long-term viral suppression that is possible with efavirenz therapy.

14. Fernandez F, Levy JK. Psychopharmacology in HIV spectrum disorders. Psychiatr Clin North Am 1994;17:135-48.

15. Schatzberg AF, DeBattista C. Phenomenology and treatment of agitation. J Clin Psychiatry 1999;60(Suppl 15):17-20.

16. Tseng AL, Foisy MM. Significant interactions with new antiretrovirals and psychotropic drugs. Ann Pharmacother 1999;33:461-73.

17. Holbrook AM, Crowther R, Lotter A, et al. The diagnosis and management of insomnia in clinical practice: A practical evidencebased approach. CMAJ 2000;162:216-20.

18. Thase ME. Treatment issues related to sleep and depression. J Clin Psychiatry 2000;61(Suppl 11):46-50.

19. Tseng AL, Foisy MM. Management of drug interactions in patients with HIV. Ann Pharmacother 1997;31:1040-50.

20. Pitscitelli SC, Flexner C, Minor JR, et al. Drug interactions in patients infected with human immunodeficiency virus. Clin Infect Dis 1996;23:685-93.

21. Sustiva Product Monograph. Mississauga: DuPont Pharma Inc, 2001.

22. Whooley MA, Simon GE. Managing depression in medical outpatients. N Engl J Med 2000;324:1942-9.

23. Rubinstein ML, Selwyn PA. High prevalence of insomnia in an outpatient population with HIV infection. J Acquir Immune Defic Syndr Hum Retrovirol 1998;19:260-5.

24. Penzak SR, Reddy YS, Grimsley SR. Depression in patients with HIV infection. Am J Health Syst Pharm 2000;57:376-86.

25. American Psychiatric Association. Diagnostic and Statistical Manual of Mental Disorders, 4th edn, text revision. Washington: American Psychiatric Association, 2000.

26. Elliott AJ, Russo J, Bergam K, et al. Antidepressant efficacy in HIVseropositive outpatients with major depressive disorder: An open trial of nefazodone. J Clin Psychiatry 1999;60:226-31.

27. Ferrando SJ, Goldman JD, Charness WE. Selective serotonin reuptake inhibitor treatment of depression in symptomatic HIV infection and AIDS. Improvements in affective and somatic symptoms. Gen Hosp Psychiatry 1997;19:89-97.

28. Rabkin JG, Rabkin R, Harrison W, et al. Effect of imipramine on mood and enumerative measures of immune status in depressed patients with HIV illness. Am J Psychiatry 1994;151:516-23.

29. Zisook S, Peterkin J, Goggin KJ, et al. Treatment of major depression in HIV-seropositive men. J Clin Psychiatry 1998;59:217-24.

30. Cole JO, Bodkin JA. Antidepressant side effects. J Clin Psychiatry 1990;51(Suppl 1):21-6.

31. Depression Guidelines Panel. Depression in Primary Care: Volume 2. Treatment of Major Depression. Clinical Practice Guideline, Number 5. AHCPR Publication No. 93-0551. Rockville: United States Department of Health and Human Services, Public Health Service, Agency for Health Care Policy and Research, 1993. 


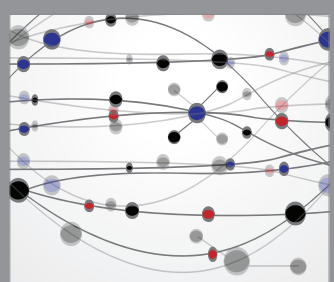

The Scientific World Journal
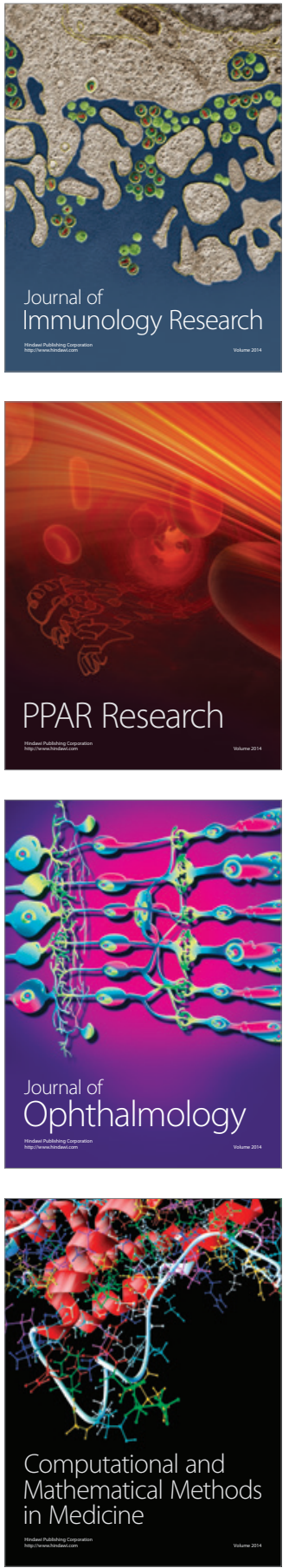

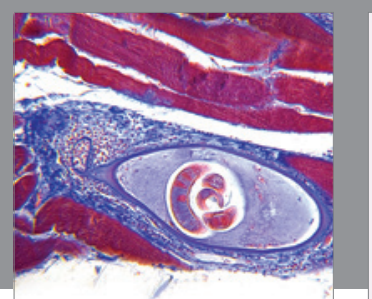

Gastroenterology Research and Practice

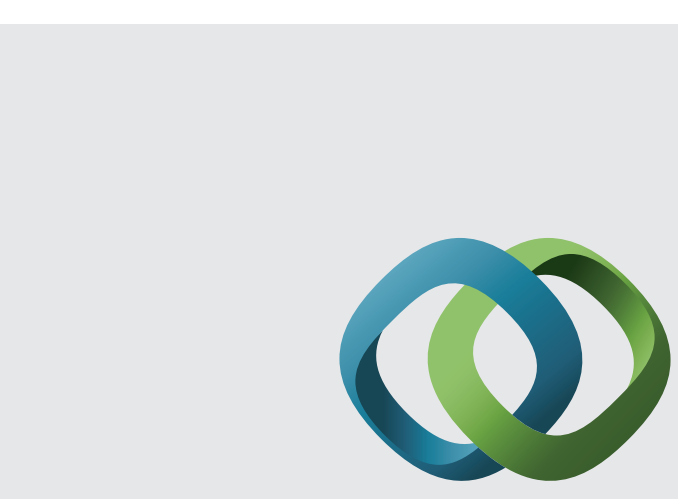

\section{Hindawi}

Submit your manuscripts at

http://www.hindawi.com
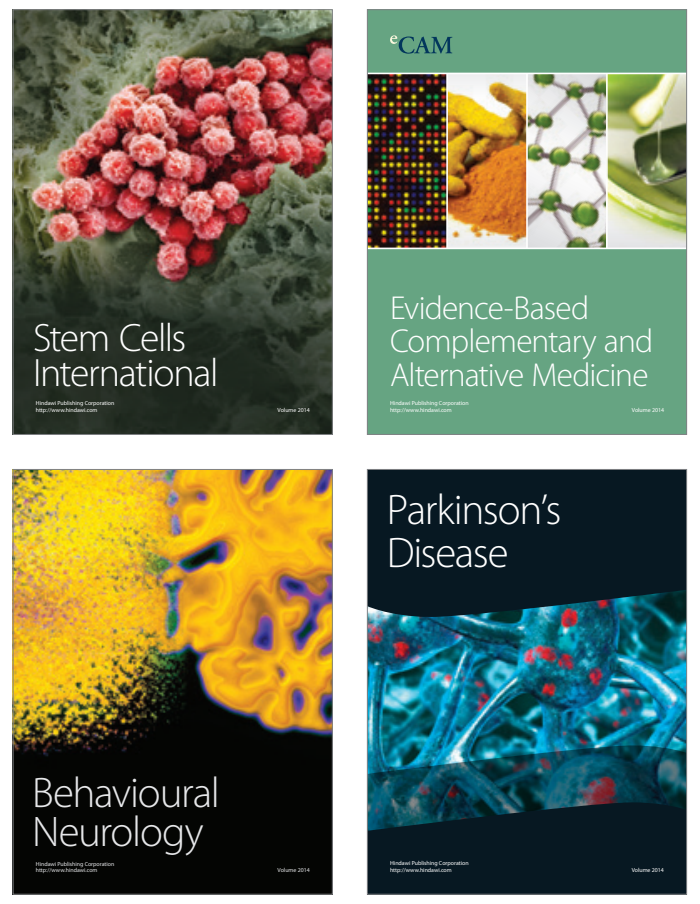
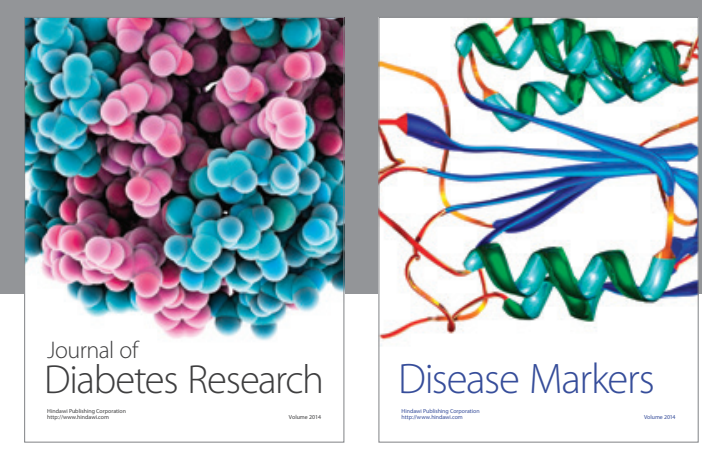

Disease Markers
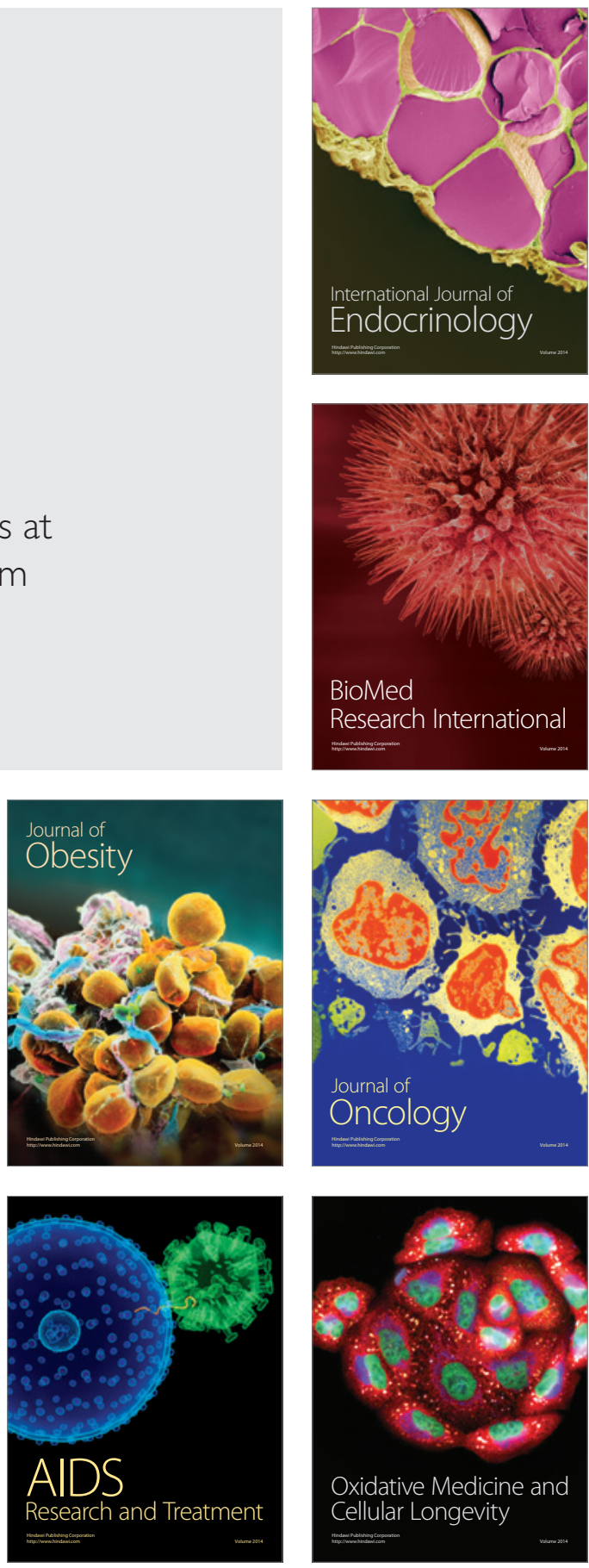